\title{
Cooperative formation of high-spin species in a photoexcited spin-crossover complex
}

\author{
Y. Moritomo \\ Department of Physics, University of Tsukuba, Tsukuba 305-8571, Japan \\ M. Kamiya and A. Nakamura \\ Department of Applied Physics, Nagoya University, Nagoya 464-8603, Japan \\ A. Nakamoto and N. Kojima \\ Graduate School of Arts and Science, University of Tokyo, Tokyo 158-8902, Japan
}

(Received 18 August 2005; published 24 January 2006)

\begin{abstract}
The dynamics of the photoinduced phase transition was investigated in a prototypical spin crossover complex, $\left[\mathrm{Fe}(\mathrm{ptz})_{6}\right]\left(\mathrm{BF}_{4}\right)_{2}(\mathrm{ptz}=1$-propyltetrazole), under photoexcitation at $77 \mathrm{~K}$ near the transition temperature $\left(T_{\mathrm{c}}=130 \mathrm{~K}\right)$. When the excitation power $I$ exceeds $\approx 1 \mathrm{~mW} / \mathrm{mm}^{2}$, we observed an "acceleration" of the creation rate of the density $n_{\mathrm{HS}}$ of the high-spin (HS) species after a characteristic incubation period. The "acceleration" is interpreted in terms of the negative pressure effect; the photocreated HS species with a larger ionic radius expands the volume to cause the structural phase transition.
\end{abstract}

DOI: 10.1103/PhysRevB.73.012103

PACS number(s): $64.90 .+\mathrm{b}, 68.18 . \mathrm{Jk}$

The photoinduced phenomena ${ }^{1}$ have been extensively investigated both from the fundamental and technical points of view. In an extreme case, the photoexcitation causes the macroscopic structural change, or the so-called photoinduced phase transition (PIPT). So far, a long list of researchers have reported on persistent PIPTs in spin-crossover complexes, ${ }^{2-7}$ mixed-valence gold complex, $\mathrm{Cs}_{2} \mathrm{Au}_{2} \mathrm{Br}_{6} \quad$ (Ref. 8) transition metal cyanides, ${ }^{9-14}$ iodine-bridged binuclear Pt compounds, ${ }^{15}$ organic radical crystal, TTTA (1,3,5-trithia-2,4,6-triazapentalenyl), ${ }^{16} \quad$ polydiacetylene crystal, $^{17}$ and so on. Most of these materials ${ }^{14-17}$ respond nonlinearly with the excitation power $I$, suggesting that cooperative interaction originated by the electron correlation and/or the electron-lattice coupling plays a key role at the PIPT process. For example, Koshino and Ogawa ${ }^{18}$ theoretically proposed domino effects in a one-dimensional system; a photoinduced local structural change causes a global structural change when the intersite interaction is short-ranged and moderately strong. Thus, the clarification of the dynamical process of the PIPT is one of the hottest topics in solid state physics.

Among the materials that show PIPT, the spin crossover complex consists of the divalent $\mathrm{Fe}^{2+}$ ions surrounded by the ligand molecules and the countercations. This compound was most intensively investigated ${ }^{19-21}$ in the past few decades due to the following reasons. First, the role of photoexcitation is quite simple; the photonexcitation converts the lowspin $\left(\mathrm{LS} ; t_{2 \mathrm{~g}}^{6}\right) \mathrm{Fe}^{2+}$ site into the high-spin (HS; $t_{2 \mathrm{~g}}^{4} e_{\mathrm{g}}^{2}$ ) state via an intersystem crossing. Secondly, the density $n_{\mathrm{HS}}$ of the HS species is easily estimated from the dipole-forbidden transition of the LS $\mathrm{Fe}^{2+}$ ion. Recently, Ogawa et al. ${ }^{19}$ have investigated the dynamical behavior of $n_{\mathrm{HS}}$ of $\left[\mathrm{Fe}\left(2\right.\right.$-pic) $\left.{ }_{3}\right] \mathrm{Cl}_{2} \cdot \mathrm{EtOH}$ at $2.2 \mathrm{~K}$ far below the transition temperature $\left(T_{\mathrm{c}}=114-121 \mathrm{~K}\right)$ for the thermally-induced LS-HS phase transition. They reported a nonlinear increase of $n_{\mathrm{HS}}$ after an incubation period. This suggests that some cooperative interaction governs the PIPT process, ${ }^{22}$ even though the large quantum efficiency ( $\Phi \approx 34$ site/photon) was not quan- titatively explained. Contrary to this observation, Enachescu et $a l .{ }^{20}$ have carefully re-examined the Ogawa's work, and reached a different conclusion that $\Phi \sim 1$ irrespective of the excitation power $I$. They further concluded that the observed $n_{\mathrm{HS}}-t$ curve can be quantitatively reproduced by a simple rate equation, ${ }^{2}$

$$
\frac{\mathrm{d} n_{\mathrm{HS}}}{\mathrm{d} t}=I \Gamma\left(1-n_{\mathrm{HS}}\right)-n_{\mathrm{HS}} k_{0} \exp \left(-\alpha n_{\mathrm{HS}}\right),
$$

where $\Gamma, k_{0}, \alpha$ is the conversation efficiency, the bare relaxation rate and the avalanche factor, respectively. Thus, the dynamics of the PIPT process is still controversial even in the spin crossover complex.

Here, our strategy to extract the cooperative effect in the PIPT process is to enhance the intersite interaction by approximating the energy level of the metastable state to that of the ground state. For this purpose, we have investigated the PIPT process in the most prototypical spin crossover complex, $\left[\mathrm{Fe}(\mathrm{ptz})_{6}\right]\left(\mathrm{BF}_{4}\right)_{2}$, near $T_{\mathrm{c}}$ for the LS-HS phase transition. As we had expected, we observed an "acceleration" of the creation rate of the density $n_{\mathrm{HS}}$ after a characteristic incubation period when $I$ exceeds $\approx 1 \mathrm{~mW} / \mathrm{mm}^{2}$. Based on the quantitative analysis of the $n_{\mathrm{HS}^{-}} t$ curves, we propose that the photocreated HS species with a larger ionic radius induces a negative pressure to cause the structural phase transition.

Single crystals of $\left[\mathrm{Fe}(\mathrm{ptz})_{6}\right]\left(\mathrm{BF}_{4}\right)_{2}$ were prepared in the manner of the procedure described in Ref. 23. The typical size of the crystals was $4 \times 4 \times 0.05 \mathrm{~mm}^{3}$. We measured the temperature dependence of magnetic susceptibility, and confirmed that our crystal shows the thermally induced LS-HS phase transition at $T_{\mathrm{c}}=130 \mathrm{~K}$. At the phase transition, the color of the crystal changes from transparent purple (the LS phase) to transparent and colorless (the HS phase). We emphasize that the cell volume discontinuously increases by $3 \%$ at the LS-HS phase transition, ${ }^{24}$ reflecting the fact that the ionic radius $\left(r_{\mathrm{HS}}=0.78 \AA\right.$ ) of the $\mathrm{HS} \mathrm{Fe}^{2+}$ ion is larger than 

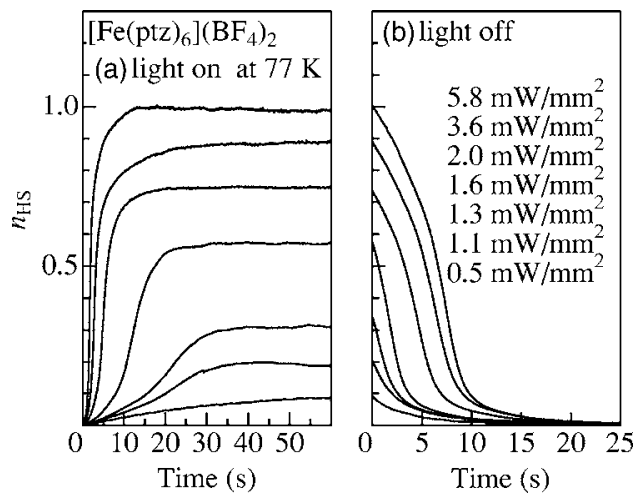

FIG. 1. Temporal behavior of the density $n_{\mathrm{HS}}$ of the high-spin (HS) species of $\left[\mathrm{Fe}(\mathrm{ptz})_{6}\right]\left(\mathrm{BF}_{4}\right)_{2}$ measured at $77 \mathrm{~K}$; (a) photoexcitation starts at $t=0$ and (b) photoexcitation stops at $t=0$.

that $\left(r_{\mathrm{LS}}=0.61 \AA\right)$ of the $\mathrm{LS} \mathrm{Fe}{ }^{2+}$ ion. The density $n_{\mathrm{HS}}$ of the HS species can be precisely estimated from the intensity of the intra-atomic absorption of the LS species located at $\approx 2.3 \mathrm{eV}$. We used an $\mathrm{Ar}^{+}$laser $(514.5 \mathrm{~nm})$ and a cw yttrium aluminum garnet (YAG) laser $(532 \mathrm{~nm})$ as the excitation and probe light sources, respectively. The intensity of the excitation light was continuously controlled with a circular neutral density filter. The excitation light illuminated the sample so that the excitation light $(3 \mathrm{~mm} \phi)$ completely overlaps the probe light $(1 \mathrm{~mm} \phi)$. The intensity of the transmitted probe light was digitally recorded as a function of time. In order to make the measurement efficient, the magnitude of $I$ as well as the on/off action of the excitation light was controlled by a personal computer. We further measured the temperature at the sample surface under photoexcitation, and confirmed that the temperature rise is less than $2 \mathrm{~K}$ even at the maximum excitation condition $\left(I=7.0 \mathrm{~mW} / \mathrm{mm}^{2}\right)$.

We show in Fig. 1(a) the prototypical $n_{\mathrm{HS}}-t$ curves of $\left[\mathrm{Fe}(\mathrm{ptz})_{6}\right]\left(\mathrm{BF}_{4}\right)_{2}$ measured at $77 \mathrm{~K} . n_{\mathrm{HS}}=1$ means that all the $\mathrm{Fe}^{2+}$ sites take the HS state. In the strong excitation condition (for example, see the $2.0 \mathrm{~mW} / \mathrm{mm}^{2}$ data), $n_{\mathrm{HS}}$ superlinearly increases with time, and then becomes almost constant. Hereafter, we describe this constant value as $\left\langle n_{\mathrm{HS}}\right\rangle$. We note that $\left\langle n_{\mathrm{HS}}\right\rangle$ is not necessarily in unity in the photoexcited steady state, because the photocreation process of the HS species balances with their thermal relaxation processes of them. Actually, the relaxation speed [see Fig. 1(b)] is comparable with the creation speed. This makes a sharp contrast with the low temperature experiment reported by Enachescu et al. ${ }^{20}$ in which $n_{\mathrm{HS}}$ seems to approach unity in every excitation condition (see Fig. 2 of Ref. 20). In the intermediated case (for example, see the $1.6 \mathrm{~mW} / \mathrm{mm}^{2}$ data), $n_{\mathrm{HS}}$ nonlinearly increases after the characteristic incubation period, and then becomes constant. In Fig. 2, we show the microscope image of the $\left[\mathrm{Fe}(\mathrm{ptz})_{6}\right]\left(\mathrm{BF}_{4}\right)_{2}$ single crystal taken at one second intervals after the photoexcitation of $1.7 \mathrm{~mW} / \mathrm{mm}^{2}$. The color shows negligible change until $3 \mathrm{~s}$ after the photoexcitation [Fig. 2(a)-2(c)], but drastically brightens up at $t=4 \mathrm{~s}$ [Fig. 2(d)] and $t=5 \mathrm{~s}$ [Fig. 2(e)]. Such an incubation behavior implies that some cooperative interaction dominates the PIPT process.

In Fig. 3, we plotted $\left\langle n_{\mathrm{HS}}\right\rangle$ in the photoexcited steady state
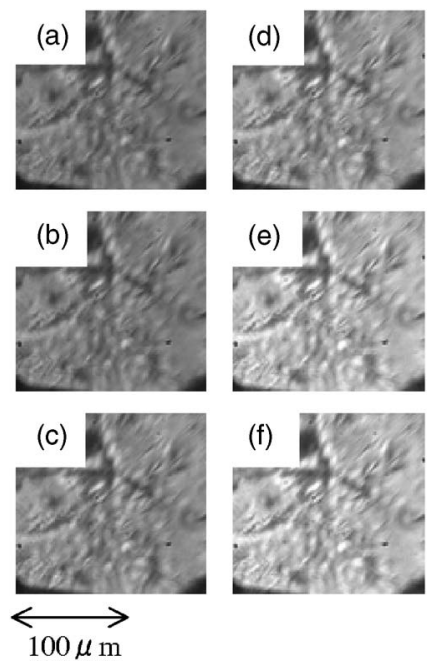

FIG. 2. Microscope image of a $\left[\mathrm{Fe}(\mathrm{ptz})_{6}\right]\left(\mathrm{BF}_{4}\right)_{2}$ single crystal at $80 \mathrm{~K}$. The pictures were taken at one second intervals after the photoexcitation of $1.7 \mathrm{~mW} / \mathrm{mm}^{2}$; (a) $1 \mathrm{~s}$, (b) $2 \mathrm{~s}$, (c) $3 \mathrm{~s}$, (d) $4 \mathrm{~s}$, (e) $5 \mathrm{~s}$, and (f) $6 \mathrm{~s}$. The dark (bright) color corresponds the LS (HS) state.

against $I$. The increasing (decreasing) speed of each $I$-step was about $1 \mathrm{~s}$. The magnitudes of $\left\langle n_{\mathrm{HS}}\right\rangle$ were measured $30 \mathrm{~s}$ after the increase (decrease) of $I$. In the weak excitation region $\left(I<1 \mathrm{~mW} / \mathrm{mm}^{2}\right),\left\langle n_{\mathrm{HS}}\right\rangle$ increases linearly with $I$, reflecting the fact that the creation rate of the HS species is proportional to $I$. With further increases of $I$ beyond $\approx 1 \mathrm{~mW} / \mathrm{mm}^{2}$, however, $\left\langle n_{\mathrm{HS}}\right\rangle$ steeply increases to reach $\sim 1$. We interpreted this threshold behavior in terms of the dynamical phase transition ${ }^{21}$ of the photoexcited steady state from the $\left\langle n_{\mathrm{HS}}\right\rangle \approx 0$ state $\left(I \leqslant 2 \mathrm{~mW} / \mathrm{mm}^{2}\right)$ to the $\left\langle n_{\mathrm{HS}}\right\rangle \approx 1\left(I \geqslant 2 \mathrm{~mW} / \mathrm{mm}^{2}\right)$ state. We further observed optical hysteresis $^{26}$ between the $I$-increasing run and the $I$-decreasing run, perhaps due to the significant structural change at the PIPT of $\left[\mathrm{Fe}(\mathrm{ptz})_{6}\right]\left(\mathrm{BF}_{4}\right)_{2} .{ }^{25}$

Now, let us qualitatively analyze the experimentally obtained $n_{\mathrm{HS}}-T$ curve (see Fig. 1) with a simple rate equation [Eq. (1)]. The first term, $I \Gamma\left(1-n_{\mathrm{HS}}\right)$, represents the photocre-

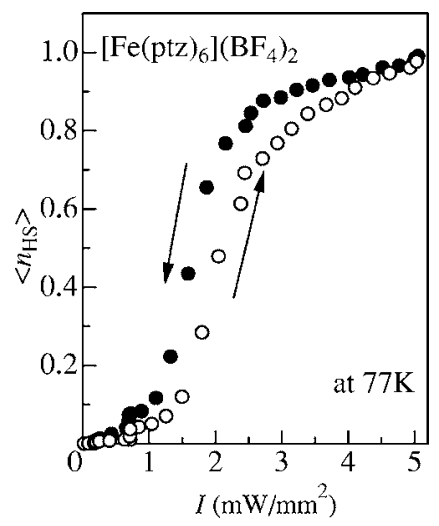

FIG. 3. Density $\left\langle n_{\mathrm{HS}}\right\rangle$ of the HS species in the photoexcited steady state of $\left[\mathrm{Fe}(\mathrm{ptz})_{6}\right]\left(\mathrm{BF}_{4}\right)_{2}$ at $77 \mathrm{~K}$ against the excitation power $I$. Open (closed) circles were obtained in the $I$-increasing ( I-decreasing) run. 

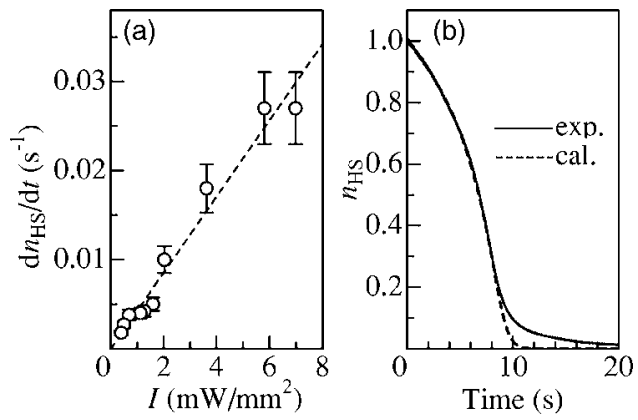

FIG. 4. (a) Initial creation rate of $n_{\mathrm{HS}}$ of $\left[\mathrm{Fe}(\mathrm{ptz})_{6}\right]\left(\mathrm{BF}_{4}\right)_{2}$ at 77 $\mathrm{K}$ against the excitation power $I$. A straight line stands for the result of the least-square-fitting. (b) Relaxation curve of $n_{\mathrm{HS}}$ after photoexcitation stops $\left(I=5.8 \mathrm{~mW} / \mathrm{mm}^{2}\right)$ together with the calculation (broken curve) based on the rate equation.

ation process of the HS species. We plotted in Fig. 4(a) the initial creation rate $\mathrm{d} n_{\mathrm{HS}} / \mathrm{d} t$ at $n_{\mathrm{HS}}=0 . \mathrm{d} n_{\mathrm{HS}} / \mathrm{d} t$ linearly increases with $I$, indicating that the initial stage is dominated by the photocreation process of the HS species. The conversion efficiency $\Gamma$ was estimated at $4 \times 10^{-3} \mathrm{~mW} / \mathrm{mm}^{2} / \mathrm{s}$ from the slope of the data points. With use of the absorption coefficient $\left(\alpha=60 \mathrm{~cm}^{-1}\right)$ at $532 \mathrm{~nm}$ and $\Gamma$, the quantum efficiency $\Phi$ becomes 0.3 site/photon. ${ }^{27}$ On the other hand, the second term, $n_{\mathrm{HS}} k_{0} \exp \left(-\alpha n_{\mathrm{HS}}\right)$, represents the relaxation process of the HS species, in which the interaction between the HS species is phenomenologically included as the suppression of the relaxation rate in the HS-rich region. ${ }^{2}$ By the fitting of the relaxation curve [see Fig. 4(b)], the bare relaxation rate $k_{0}$ and the avalanche factor $\alpha$ are determined to be $2 \mathrm{~s}^{-1}$ and 3.9, respectively. Here, note that this simple meanfield picture of the rate equation cannot include the actual spatial fluctuation. In this sense, the above-obtained parameters were the effective (or weighted averaged) ones. Incidentally, the notable deviation between the experiment and the calculation $(t \geqslant 8 \mathrm{~s})$ is due to the spatial fluctuation of $n_{\mathrm{HS}} \cdot{ }^{28}$

The rate equation with the effective parameters, that is, $\Gamma=4 \times 10^{-3} \mathrm{~mW} / \mathrm{mm}^{2} / \mathrm{s}, k_{0}=2 \mathrm{~s}^{-1}$, and $\alpha=3.9$, cannot reproduce the overall temporal behavior of $n_{\mathrm{HS}}$. This is because the creation rate $I \Gamma$ [order of $0.01 \mathrm{~s}^{-1}$; see Fig. 4(a)] is too slow to reproduce the experimentally-observed fast increase of $n_{\mathrm{HS}}$ [order of $10 \mathrm{~s}$; see Fig. 1(a)], even if we neglect the relaxation process. In contrast, the PIPT process of $\left[\mathrm{Fe}(2-\mathrm{pic})_{3}\right] \mathrm{Cl}_{2} \cdot \mathrm{EtOH}$ at a low temperature ${ }^{20}$ is more gradual, and can be quantitatively reproduced by the same rate equation [Eq. (1)]. We, therefore, conclude that an extra creation process of the HS species dominates the present PIPT process near $T_{\mathrm{c}}$, where the HS state and the LS ground state are nearly degenerate.

Spiering and co-workers have systematically investigated the second coordination effect by a chemical substitution in $\left[\mathrm{Fe}(2-\mathrm{pic})_{3}\right] \mathrm{Cl}_{2} \cdot \mathrm{EtOH}^{30-32}$ and $\left[\mathrm{Fe}(\mathrm{ptz})_{6}\right]\left(\mathrm{BF}_{4}\right)_{2} \cdot{ }^{33}$ They have found that the chemical substitution significantly modifies the LS-HS transudation temperature, and have ascribed the chemical impurity effect to the strain effect. ${ }^{34}$ For example, $T_{\mathrm{c}}$ of $\left[\mathrm{Fe}_{1-x} \mathrm{Zn}_{x}(\mathrm{ptz})_{6}\right]\left(\mathrm{BF}_{4}\right)_{2}$ decreases in proportion to the concentration $x$ of the $\mathrm{Zn}^{2+}$ impurity as

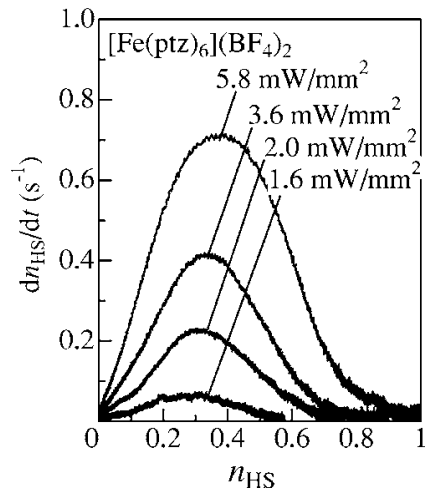

FIG. 5. Creation rate of $n_{\mathrm{HS}}$ of $\left[\mathrm{Fe}(\mathrm{ptz})_{6}\right]\left(\mathrm{BF}_{4}\right)_{2}$ at $77 \mathrm{~K}$ against $n_{\mathrm{HS}}$ at several excitation conditions.

$T_{\mathrm{c}} \approx 130-30 x .^{33}$ Here, note that the ionic radius $\left(r_{\mathrm{Zn}}=0.74 \AA\right)$ of the $\mathrm{Zn}^{2+}$ ion is larger than that $\left(r_{\mathrm{LS}}=0.61 \AA\right)$ of the $\mathrm{LS} \mathrm{Fe}^{2+}$ ion, and hence, the $\mathrm{Zn}^{2+}$ impurities expand the lattice to stabilize the HS phase with larger cell volume. We think that a similar strain is induced by the photocreated HS species, because its ionic radius $\left(r_{\mathrm{HS}}=0.78 \AA\right)$ is larger than that $\left(r_{\mathrm{LS}}=0.61 \AA\right)$ of the $\mathrm{LS} \mathrm{Fe}{ }^{2+}$ ion. Then, the photocreated HS species also suppress $T_{\mathrm{C}}$ via the stress effect, analogously to the case of the $\mathrm{Zn}^{2+}$ impurity. In other words, the photocreated HS species induces the negative pressure within the system. In an extreme case, the system would reach the phase boundary between the LS phase and the HS phase, and show the structural phase transition. ${ }^{29}$ In this picture, the present PIPT process can be described as follows: the HS species gradually accumulate by the photocreation process in the incubation period, and then, the system shows the phase transition into the $\left\langle n_{\mathrm{HS}}\right\rangle \approx 1$ state when $n_{\mathrm{HS}}(t)$ reaches a critical value.

The above-mentioned physical impurity effect is apparently analogous to the domino effect ${ }^{18}$ on the point that the photoinduced local structural change causes a global structural change. The model, however, is based on the onedimensional system, and is not applicable to the present three-dimensional system. In addition, the condition that a single photocreated structural change causes a global structural change becomes more severe in the high-dimensional system due to the surface energy loss at the phase boundary. In contrast, we consider that the aggregate of the photocreated HS species modify the energy of the metastable HS phase relative to the ground LS phase, and eventually exchange the two energy levels to cause a global phase transition.

In Fig. 5, we show the creation rate $\mathrm{d} n_{\mathrm{HS}} / \mathrm{d} t$ against $n_{\mathrm{HS}}$ at several excitation conditions. We found that the $\mathrm{d} n_{\mathrm{HS}} / \mathrm{d} t-n_{\mathrm{HS}}$ curves show broad maxima at $n_{\mathrm{HS}} \approx 0.3$, irrespective of $I$. [Note that the scale of the horizontal axis of Fig. 5 is two orders larger than that of Fig. 4.] This suggests that the critical density, where the system reaches the phase boundary, is located at $\approx 0.3$. Here, we emphasize that the physical impurity has a dynamic nature, and migrates in the crystal via the creation/relaxation process. Then, the HS-rich regions ${ }^{35}$ are spontaneously created in the crystal due to the intrinsic spatial fluctuation of $n_{\mathrm{HS}}$. Such HS-rich regions 
could show the phase transition before the spatial average of $n_{\mathrm{HS}}$ reaches the critical value. This may be the origin for the gradual increase of $\mathrm{d} n_{\mathrm{HS}} / \mathrm{d} t$, as observed in Fig. 5. We, however, cannot observe any trace of the spatial fluctuation in the microscopic images (see Fig. 2), whose spatial resolution is $\sim 10 \mu \mathrm{m}$. This suggests that the size of the spatial fluctuation is much less than $\sim 10 \mu \mathrm{m}$.

In summary, we have systematically investigated the temporal behavior of $n_{\mathrm{HS}}$ of the photoexcited $\left[\mathrm{Fe}(\mathrm{ptz})_{6}\right]\left(\mathrm{BF}_{4}\right)_{2}$ near $T_{\mathrm{c}}$ for the LS-HS phase transition, as a function of excitation power $I$. We observed an acceleration of the creation rate $\mathrm{d} n_{\mathrm{HS}} / \mathrm{d} t$ after the characteristic incubation period when $I$ exceeds $\approx 1 \mathrm{~mW} / \mathrm{mm}^{2}$. We interpreted the acceleration in terms of the negative pressure effect induced by the photocreated HS species.

This work was supported by a Grant-in-Aid for Scientific Research from the Ministry of Education Science, Sports and Culture of Japan and from the Yazaki Foundation. The authors thank Dr. T. Kawamoto and Prof. T. Ogawa for fruitful discussions.
${ }^{1}$ Relaxation of Excited States and Photoinduced Structural Transitions, edited by K. Nasu (Springer-Verlag, Berlin, 1997).

${ }^{2}$ A. Hauser, J. Jeftic, H. Romstedt, R. Hinek, and H. Spiering, Coord. Chem. Rev. 190-192, 471 (1999).

${ }^{3}$ S. Decurtins, P. Gütlich, K. M. Hasselbach, A. Hauser, and H. Spiering, Inorg. Chem. 24, 2174 (1985).

${ }^{4}$ A. Hauser, P. Gütlich, and H. Spiering, Inorg. Chem. 25, 4245 (1986)

${ }^{5}$ A. Hauser, Chem. Phys. Lett. 124, 543 (1986).

${ }^{6}$ N. O. Moussa, G. Molnar, S. Bonhonmmeau, A. Zwick, S. Mouri, K. Tanaka, J. A. Real, and A. Boussekson, Phys. Rev. Lett. 94, 107205 (2005).

${ }^{7}$ T. Tayagaki and K. Tanaka, Phys. Rev. Lett. 86, 2886 (2001).

${ }^{8}$ X. J. Liu, Y. Moritomo, M. Ichida, A. Nakamura, and N. Kojima, Phys. Rev. B 61, 20 (2000)

${ }^{9}$ O. Sato, T. Iyoda, A. Fujishima, and K. Hashimoto, Science 272, 704 (1996).

${ }^{10}$ O. Sato, Y. Einaga, A. Fujishima, and K. Hashimoto, Inorg. Chem. 38, 4405 (1999).

${ }^{11}$ Y. Moritomo, M. Hanawa, Y. Ohishi, K. Kato, M. Takata, A. Kuriki, E. Nishibori, M. Sakata, S. Ohkoshi, H. Tokoro, and K. Hashimoto, Phys. Rev. B 68, 144106 (2003).

${ }^{12}$ M. Hanawa, Y. Moritomo, A. Kuriki, J. Tateishi, K. Kato, M. Takata, and M. Sakata, J. Phys. Soc. Jpn. 72, 987 (2003).

${ }^{13}$ N. Shimamoto, S. Ohkoshi, O. Sato, and K. Hashimoto, Inorg. Chem. 41, 678 (2002).

${ }^{14}$ H. Tokoro, T. Matsuda, K. Hashimoto, and S. Ohkoshi, J. Appl. Phys. 97, 10M5083 (2005).

${ }^{15}$ H. Matsuzaki, T. Matsuoka, H. Kishida, K. Takizawa, H. Miyasaka, K. Sugiura, M. Yamashita, and H. Okamoto, Phys. Rev. Lett. 90, 046401 (2003).

${ }^{16}$ H. Matsuzaki, W. Fujita, K. Awaga, and H. Okamoto, Phys. Rev. Lett. 91, 017403 (2003).

${ }^{17}$ S. Koshihara, Y. Tokura, K. Takeda, and T. Koda, Phys. Rev. Lett. 68, 1148-1151 (1992).

${ }^{18}$ K. Koshino and T. Ogawa, J. Phys. Soc. Jpn. 67, 2173 (1998).

${ }^{19}$ Y. Ogawa, S. Koshihara, K. Koshino, T. Ogawa, C. Urano, and H.
Takagi, Phys. Rev. Lett. 84, 3181 (2000).

${ }^{20}$ C. Enachscu, U. Oetliker, and A. Hauser, J. Phys. Chem. B 106, 9540 (2002).

${ }^{21}$ X. J. Liu, Y. Moritomo, T. Kawamoto, A. Nakamoto, and N. Kojima, J. Phys. Soc. Jpn. 72, 1615 (2003).

${ }^{22}$ K. Koshino and T. Ogawa, J. Phys. Soc. Jpn. 68, 2164 (1999).

${ }^{23}$ P. L. Franke, J. G. Haasnoot, and A. P. Zuur, Inorg. Chim. Acta 59, 5 (1982).

${ }^{24}$ Y. Moritomo, K. Kato, A. Nakamoto, N. Kojima, E. Nishibori, M. Takata, and M. Sakata, J. Phys. Soc. Jpn. 71, 1015 (2002).

${ }^{25}$ Y. Moritomo, K. Kato, A. Kuriki, A. Nakamoto, N. Kojima, M. Takata, and M. Sakata, J. Phys. Soc. Jpn. 71, 2609 (2002).

${ }^{26}$ J. Jeftic et al., Polyhedron 20, 1599 (2001).

${ }^{27}$ The quantum efficiency ( $\Phi=0.3$ sites/photon) is different from the value ( $=0.8$ sites/photon) reported in Ref. 34 . This is because we have derived $\Phi$ from the initial creation ratio $\mathrm{d} n_{\mathrm{HS}} / \mathrm{d} t$ at $n_{\mathrm{HS}}=0$, not from the fitting of the overall $n_{\mathrm{HS}}-t$ behavior. We emphasize that the quantum efficiency $(\Phi=13$ sites/photon) would become an unphysical value, if we reproduced the magnitude of $\left\langle n_{\mathrm{HS}}\right\rangle$ within the frame of Eq. (1).

${ }^{28}$ H. Romstedt, A. Hauser, and H. Spiering, J. Phys. Chem. Solids 59, 265 (1998).

${ }^{29}$ O. Sakai, M. Ishii, T. Ogawa, and K. Koshino, J. Phys. Soc. Jpn. 71, 2052 (2002).

${ }^{30}$ H. Köppen, E. W. Müller, C. P. Köhler, H. Spiering, E. Meissner, and P. Gütlich, Chem. Phys. Lett. 91, 348 (1982).

${ }^{31}$ R. Jakobi, H. Spiering, and P. Gütlich, J. Phys. Chem. Solids 53, 267 (1992).

${ }^{32}$ A. Vef, U. Manthe, P. Gütlich, and A. Hauzer, J. Chem. Phys. 101, 9326 (1994).

${ }^{33}$ J. Jung, G. Schmitt, L. Wiehl, A. Hauser, K. Knorr, H. Spiering, and P. Gütlich, Z. Phys. B: Condens. Matter 100, 523 (1996).

${ }^{34}$ A. Hauser, Top. Curr. Chem. 234, 155 (2004).

${ }^{35} \mathrm{We}$ observed no trace of the spatial fluctuation of $n_{\mathrm{HS}}$ in our microscope image. This suggests that the size of the fluctuation would be less than few $\mu \mathrm{m}$. 